\title{
REGIONS OF VARIABILITY FOR UNIVALENT FUNCTIONS
}

\author{
PETER DUREN AND AYŞENUR ÜNAL
}

\begin{abstract}
Let $S$ be the standard class of univalent functions in the unit disk, and let $S_{0}$ be the class of nonvanishing univalent functions $g$ with $g(0)=1$. It is shown that the regions of variability $\left\{g(r): g \in S_{0}\right\}$ and $\left\{\left(1-r^{2}\right) f^{\prime}(r): f \in S\right\}$ are very closely related but are not quite identical.
\end{abstract}

Let $S$ be the class of functions $f$ analytic and univalent in the unit disk $\mathbf{D}$, with $f(0)=0$ and $f^{\prime}(0)=1$. Let $S_{0}$ be the class of analytic univalent functions $g$ for which $g(z) \neq 0$ in $\mathbf{D}$ and $g(0)=1$. In this paper we compare the regions of variability

$$
W(\zeta)=\left\{f^{\prime}(\zeta): f \in S\right\} \quad \text { and } \quad W_{0}(\zeta)=\left\{g(\varsigma): g \in S_{0}\right\}
$$

at a fixed point $\zeta \in \mathbf{D}$. Both regions depend only on $|\zeta|$, so it suffices to consider $W(r)$ and $W_{0}(r)$ for $0<r<1$.

Initially there is no reason to expect the regions $W(r)$ and $W_{0}(r)$ to be related at all. There is only the superficial connection suggested by the nonvanishing of the derivative of a univalent function. However, we shall offer persuasive evidence in support of the conjecture that

$$
W_{0}(r)=\left(1-r^{2}\right) W(r), \quad 0 \leq r<1 .
$$

For convenience, we let $\tilde{W}(r)=\left(1-r^{2}\right) W(r)$. In $\S 1$ we motivate the conjecture that $W_{0}(r)=\tilde{W}(r)$, and we present some analytical evidence in its favor. In $\S 2$ we show how the boundaries of the two regions can be calculated, and we display samples of numerical data which seem to leave little doubt that the conjecture is true. In $\S 3$ we apply a variational method to prove that $W_{0}(r) \subset \tilde{W}(r)$. Then comes the surprise: we show in $\S 4$ that the conjecture is false. Thus although the two regions $W_{0}(r)$ and $\tilde{W}(r)$ share various features and appear "numerically identical", one is in fact a proper subset of the other.

1. Analytical evidence. The sharp estimates for $\left|f^{\prime}(r)\right|$ are given by the classical distortion theorem

$$
\frac{1-r}{(1+r)^{3}} \leq\left|f^{\prime}(r)\right| \leq \frac{1+r}{(1-r)^{3}}, \quad f \in S
$$

with equality for the Koebe function $k(z)=z(1-z)^{-2}$ and its rotation $-k(-z)$. The corresponding inequality for $S_{0}$ is $[\mathbf{1 3}, \mathbf{8}]$

$$
\left(\frac{1-r}{1+r}\right)^{2} \leq|g(r)| \leq\left(\frac{1+r}{1-r}\right)^{2}, \quad g \in S_{0}
$$

Received by the editors April 5, 1985. Presented to the American Mathematical Society in San Diego, California, on November 10, 1984.

1980 Mathematics Subject Classification. Primary 30C55; Secondary 30C70.

Key words and phrases. Univalent functions, regions of variability, extremal problems, variational methods, quadratic differentials, algebraic curves. 
with equality for $k_{0}(z)=[(1+z) /(1-z)]^{2}$ and $k_{0}(-z)$. Thus the factor $\left(1-r^{2}\right)$ converts the first pair of bounds to the second, and the points of maximum and minimum moduli for $W_{0}(r)$ and $\tilde{W}(r)=\left(1-r^{2}\right) W(r)$ are the same.

The two regions also have the same maximum and minimum arguments for $r \leq 1 / \sqrt{2}$. The well-known rotation theorem for the class $S$ (see [7, p. 99]) gives the sharp bound

$$
\left|\arg f^{\prime}(r)\right| \leq 4 \sin ^{-1} r, \quad 0 \leq r \leq 1 / \sqrt{2},
$$

with equality occurring only for certain rational functions which map $\mathbf{D}$ onto the complement of a half-line. The corresponding result for $S_{0}$ is $[\mathbf{1 1}, \mathbf{1 2}, \mathbf{9}]$

$$
|\arg g(r)| \leq 4 \sin ^{-1} r, \quad 0 \leq r \leq 1 / \sqrt{2} .
$$

(An independent proof is given in §3.) In fact, a straightforward calculation based on the known form of the extremal functions shows that the points of maximum and minimum arguments for $W_{0}(r)$ and $\tilde{W}(r)$ are precisely the same for $r \leq 1 / \sqrt{2}$. (More details are given at the end of $\S 3$.) Thus the boundaries of the two regions actually meet at four points.

Finally, we observe that the two regions have the same symmetries. Since both $S$ and $S_{0}$ are preserved under conjugation, the regions $W(r)$ and $W_{0}(r)$ are both symmetric with respect to the real axis. The other symmetry is less obvious, but it is easy to show (see $[\mathbf{1 0}$, p. 263]) that the region $\log W(r)$ is symmetric with respect to the vertical line $x=-\log \left(1-r^{2}\right)$. Thus $\log \tilde{W}(r)$ is symmetric with respect to the imaginary axis. That $\log W_{0}(r)$ has the same symmetry is evident from the simple fact that $g \in S_{0}$ implies $1 / g \in S_{0}$.

2. Numerical evidence. Several approaches have been taken to describe the logarithmic region

$$
\mathcal{L}(r)=\log W(r)=\left\{\log f^{\prime}(r): f \in S\right\} .
$$

Aleksandrov and Kopanev [3, 2] recently applied a novel form of Loewner's method to obtain an explicit parametric representation of the boundary curve. The formula is complicated but readily amenable to numerical calculation. For $0<r \leq 1 / \sqrt{2}$ it has the form

$$
\begin{aligned}
w= & -\log \left(1-r^{2}\right)+2 \tan ^{-1}\left\{\frac{b(1-c)}{\cos \theta}\right\} \\
& -\left(e^{i \theta}-i\right) \tan ^{-1}\left\{\frac{b(1-c)}{1-\sin \theta}\right\}-\left(e^{i \theta}+i\right) \tan ^{-1}\left\{\frac{b(1-c)}{1+\sin \theta}\right\} \\
& -e^{i \theta} \log \frac{1-b c}{1+b c}-\frac{1}{2} \log \frac{(b-\cos \theta)^{2}-1}{(b+\cos \theta)^{2}-1} \\
& +i \log \frac{b-\sin \theta}{b+\sin \theta}-\pi\left(\operatorname{sgn} \cos \theta-e^{i \theta}\right),
\end{aligned}
$$

where $\theta$ is a parameter with $0 \leq \theta<2 \pi$, the number $c(0<c<1)$ is the smallest positive root of the cubic equation

$$
\sin ^{2} \theta\left[\left(1-r^{2}\right) x^{3}-\left(2-r^{2}\right) x^{2}\right]+\left(1+r^{2}\right) x-r^{2}=0,
$$

and

$$
b=\left\{\left(1-c \sin ^{2} \theta\right) / c(1-c)\right\}^{1 / 2} .
$$


For $1 / \sqrt{2}<r<1$ the boundary consists of two symmetric arcs of this form, joined by two horizontal line segments.

With the help of an Apple II computer, Ünal [17] exponentiated the curve (1) to give a numerical description of the boundary of $W(r)$ for various values of $r$. The results indicate that $W(r)$ is a convex region for $r \leq \rho$, where $.245<\rho<.25$. For $r>\rho$ the minimum real part in $W(r)$ is attained at exactly two different (complex conjugate) points. The corresponding problem for the region $W_{0}(r)$ was previously treated by Duren and Schober $[8,9]$, who found an analogous critical radius $\rho_{0} \approx .249$. These results suggest the possibility that $\rho=\rho_{0}$.

On the other hand, the boundary of $W_{0}(r)$ is described by the elliptic modular function $\lambda(\tau)$, normalized so that $\lambda(0)=1, \lambda(1)=\infty$, and $\lambda(\infty)=0$. This function has a fundamental region $\Omega$ which lies in the upper half-plane above the circle $\left|\tau-\frac{1}{2}\right|=\frac{1}{2}$ and between the vertical lines $\operatorname{Re}\{\tau\}=0,1$. It is well known (see $[1$, p. 273]) that $\lambda$ maps $\Omega$ conformally onto the upper half-plane. The modular function $\mu=1 /(1-\lambda)$ has the same property, with $\mu(0)=\infty, \mu(1)=0$, and $\mu(\infty)=1$. If $\mu$ is initially defined only in $\Omega$, it may be continued analytically by Schwarz reflection to the entire upper half-plane.

The complete elliptic integral of the first kind is

$$
K(r)=\int_{0}^{1}\left[\left(1-x^{2}\right)\left(1-r^{2} x^{2}\right)\right]^{-1 / 2} d x, \quad 0<r<1 .
$$

The region $W_{0}(r)$ can now be described as follows $[15,11,12,14]$.

THEOREM A. $W_{0}(r)=\{\mu(\tau): \operatorname{Im}\{\tau\} \geq v(r)\}$, where

$$
v(r)=K\left(\sqrt{1-r^{2}}\right) / 2 K(r)
$$

It is easy to see that $v(r)$ decreases as $r$ increases, and that $v(1 / \sqrt{2})=1 / 2$. For $r<1 / \sqrt{2}$, the boundary of $W_{0}(r)$ is a simple closed curve whose upper half has the parametric representation $w=\mu(t+i v(r)), 0 \leq t \leq 1$. For $r \geq 1 / \sqrt{2}$, the boundary curve has one or more self-intersections on the real axis.

The calculation of the elliptic modular function is facilitated by its representation in terms of theta functions (see [4, pp. 355, 374]):

$$
\lambda(\tau)=\left\{\theta_{2}(q) / \theta_{3}(q)\right\}^{4}, \quad q=e^{i \pi \tau},
$$

where

$$
\theta_{2}(q)=2 q^{1 / 4} \sum_{n=1}^{\infty} q^{n(n+1)}, \quad \theta_{3}(q)=1+2 \sum_{n=1}^{\infty} q^{n^{2}}
$$

These formulas allow the numerical calculation of the boundary of $W_{0}(r)$. Ünal [17] again used the Apple II to compute the upper outer part of the boundary curve for many different values of $r$. In each case the boundary of $W_{0}(r)$ was seen to agree closely with that of $\tilde{W}(r)$, suggesting that the two curves are actually identical. Brief tables of points on the two curves, rounded off to three decimal places, are displayed below. 


$$
\begin{array}{cc}
W_{0}(r)=\left\{g(r): g \in S_{0}\right\} & \tilde{W}(r)=\left\{\left(1-r^{2}\right) f^{\prime}(r): f \in S\right\} \\
\underline{r=0.2} & \\
\hline 2.226+i 0.218 & 2.227+i 0.214 \\
1.800+i 0.830 & 1.799+i 0.831 \\
1.464+i 0.963 & 1.465+i 0.963 \\
1.302+i 0.977 & 1.301+i 0.977 \\
1.105+i 0.954 & 1.114+i 0.956 \\
0.901+i 0.878 & 0.897+i 0.876 \\
0.699+i 0.727 & 0.699+i 0.727 \\
0.532+i 0.480 & 0.532+i 0.481 \\
0.489+i 0.360 & 0.489+i 0.360 \\
0.465+i 0.253 & 0.465+i 0.253 \\
0.446+i 0.066 & 0.446+i 0.068
\end{array}
$$

$\underline{r=0.5}$

$\begin{array}{rr}8.774+i 1.633 & 8.728+i 1.790 \\ 6.859+i 4.576 & 6.784+i 4.640 \\ 4.657+i 5.679 & 4.653+i 5.685 \\ 2.537+i 5.738 & 2.556+i 5.749 \\ 0.908+i 5.095 & 0.896+i 5.096 \\ 0.686+i 3.065 & -0.695+i 3.052 \\ -0.827+i 2.278 & -0.832+i 2.163 \\ -0.592+i 1.042 & -0.585+i 1.027 \\ -0.037+i 0.275 & -0.036+i 0.274 \\ 0.028+i 0.198 & 0.028+i 0.197 \\ 0.099+i 0.073 & 0.099+i 0.074 \\ 0.111+i 0.016 & 0.111+i 0.016\end{array}$

$\underline{r=0.8}$

$80.445+i \quad 8.335$

$80.414+i \quad 8.587$

$73.442+i 29.856$

$73.345+i 30.102$

$65.358+i 41.377$

$65.364+i 41.470$

$55.208+i 50.466$

$55.322+i 50.520$

$40.392+i 58.050$

$39.175+i 58.638$

$30.183+i 60.488$

$29.566+i 60.794$

$21.869+i 61.010$

$22.465+i 61.283$

$15.510+i 60.528$

$15.607+i 60.847$

$5.307+i 58.026$

$5.043+i 58.314$

$-2.270+i 54.526$

$-2.378+i 54.930$

$-15.151+i 43.417$

$-15.011+i 44.375$

$-22.579+i 27.002$

$-22.601+i 29.512$

$-18.432+i \quad 5.860$

$-18.453+i \quad 5.039$

$-14.332+i \quad 1.534$

$\begin{array}{ll}-14.361+i & 1.002\end{array}$ 
3. Proof of inclusion. We shall now prove that every support point of the logarithmic region

$$
\mathcal{L}_{0}(r)=\log W_{0}(r)=\left\{\log g(r): g \in S_{0}\right\}
$$

lies in the region

$$
\tilde{\mathcal{L}}(r)=\log \tilde{W}(r)=\left\{\log \left[\left(1-r^{2}\right) f^{\prime}(r)\right]: f \in S\right\} .
$$

The inclusion $\mathcal{L}_{0}(r) \subset \tilde{\mathcal{L}}(r)$ then follows from the convexity of $\mathcal{L}(r)$, hence of $\tilde{\mathcal{L}}(r)$.

The convexity of $\mathcal{L}(r)$ and the geometric properties of the extremal functions were found by Grad [10] and Pfluger [16]. They used variational methods to study the extremal problem

$$
\max _{h \in S} \operatorname{Re}\left\{e^{i \sigma} \log h^{\prime}(r)\right\}
$$

A version of Pfluger's elegant argument is presented in [17]. The results are summarized in the following theorem.

THEOREM B. The region $\mathcal{L}(r)$ is convex for $0<r<1$ and strictly convex for $r \leq 1 / \sqrt{2}$. The extremal problem (2) has a unique solution unless $e^{i \sigma}= \pm i$ and $r>1 / \sqrt{2}$. Each extremal function maps $\mathbf{D}$ onto the complement of a system $\Gamma$ of analytic arcs which are trajectories of the quadratic differential (7). There are three cases:

(i) If $e^{i \sigma}= \pm 1$, or if $e^{i \sigma}= \pm i$ and $r \leq 1 / \sqrt{2}$, then $\Gamma$ is a half-line;

(ii) If $e^{i \sigma} \neq \pm 1$ or $\pm i$, then $\Gamma$ lies on an analytic arc which spirals into either 0 or $f(r)$, where $f$ is the extremal function;

(iii) If $e^{i \sigma}= \pm i$ and $r>1 / \sqrt{2}$, then there are infinitely many extremal functions. In each instance $\Gamma$ has a fork or a corner at $f(r) / 2$.

We are now prepared to prove the inclusion theorem.

THEOREM 1. $\mathcal{L}_{0}(r) \subset \tilde{\mathcal{L}}(r)$ and so $W_{0}(r) \subset \tilde{W}(r)$ for $0<r<1$.

PROOF. Fix $r(0<r<1)$ and a point $e^{i \sigma}$ of unit modulus. By the compactness of $S_{0} \cup\{1\}$, there is a function $g \in S_{0}$ which solves the extremal problem

$$
\max _{h \in S_{0}} \operatorname{Re}\left\{e^{i \sigma} \log h(r)\right\} .
$$

Thus $\log g(r)$ is a support point of the region $\mathcal{L}_{0}(r)$, and every support point is so obtained by a suitable choice of $e^{i \sigma}$. In view of the convexity of $\tilde{\mathcal{L}}(r)$, we need only show that $\log g(r) \in \tilde{\mathcal{L}}(r)$.

The method of boundary variation, as developed for $S_{0}$ in [8], shows that $g$ maps $\mathrm{D}$ onto the complement of an analytic arc $\Gamma_{0}$ extending from 0 to $\infty$ and satisfying

$$
\frac{e^{i \sigma}\left(1-B_{0}\right)}{w(w-1)\left(w-B_{0}\right)} d w^{2}>0, \quad B_{0}=g(r) .
$$

Note that $\Gamma_{0}$ is a single unbranched arc because the quadratic differential has a simple pole at infinity and no zeros. Since $g \in S_{0}$, it is clear that $0 \in \Gamma_{0}$. Thus $\Gamma_{0}$ must terminate at the origin, because the quadratic differential has a simple pole there. 
Parametrizing $\Gamma_{0}$ by $w=g\left(e^{i t}\right)$, we conclude that

$$
R(z)=\frac{e^{i \sigma}\left(B_{0}-1\right) z^{2}\left[g^{\prime}(z)\right]^{2}}{g(z)[g(z)-1]\left[g(z)-B_{0}\right]} \geq 0
$$

for $|z|=1$. This function $R(z)$ is analytic and nonvanishing in $\mathbf{D}$ except for a simple pole at $z=r$ and a simple zero at $z=0$. On the unit circle, its only possible singularities or zeros are at the points $e^{i \alpha}$ and $e^{i \beta}$ where $g\left(e^{i \alpha}\right)=0$ and $g\left(e^{i \beta}\right)=\infty$. But $g$ has a double zero at $e^{i \alpha}$ and a double pole at $e^{i \beta}$, so it is easily seen that $R$ is analytic and nonvanishing at these points. By Schwarz reflection, $R$ can be continued analytically to the whole plane, and it has the structure $\overline{R(z)}=R(1 / \bar{z})$. The continued function has a simple pole at $1 / r$ and a simple zero at $\infty$.

It follows that $R$ is a rational function of the form

$$
R(z)=\frac{A z}{(z-r)(1-r z)},
$$

where $A$ is a positive constant. Equating the expressions (5) and (6) for $R(z)$ and letting $z$ tend to 0 , we find $A=e^{i \sigma} r g^{\prime}(0)$. Letting $z$ tend to $r$, we obtain

$$
A=e^{i \sigma} r\left(1-r^{2}\right) g^{\prime}(r) / B_{0} .
$$

Equating the two expressions for $A$, we obtain

$$
g(r)=\left(1-r^{2}\right) g^{\prime}(r) / g^{\prime}(0) .
$$

On the other hand, the function $f=(g-1) / g^{\prime}(0)$ belongs to $S$ and $f^{\prime}(r)=$ $g^{\prime}(r) / g^{\prime}(0)$. Thus $g(r)=\left(1-r^{2}\right) f^{\prime}(r)$ for some $f \in S$, and we have proved that $\log g(r) \in \tilde{\mathcal{L}}(r)$. This completes the proof.

COROLlary. For each $g \in S_{0}$,

$$
|\arg g(z)| \leq \begin{cases}4 \sin ^{-1} r, & r=|z| \leq \frac{1}{\sqrt{2}}, \\ \pi+\log \frac{r^{2}}{1-r^{2}}, & \frac{1}{\sqrt{2}}<r<1 .\end{cases}
$$

This bound is sharp for each $r \leq 1 / \sqrt{2}$ but not otherwise.

PROOF. The bound is a direct consequence of Theorem 1 and the rotation theorem for the class $S$. For $r \leq 1 / \sqrt{2}$ the bound is attained by the function

$$
G(z)=\left(\frac{1+i e^{i \alpha} z}{1-i e^{-i \alpha} z}\right)^{2}, \quad \alpha=\sin ^{-1} r .
$$

A calculation shows that $\arg G(r)=4 \sin ^{-1} r$ and that $G(r)=\left(1-r^{2}\right) F^{\prime}(r)$, where $F=(G-1) / G^{\prime}(0) \in S$. Thus

$$
F(z)=\frac{z-r z^{2}}{\left(1-i e^{-i \alpha} z\right)^{2}} .
$$

In particular, this shows that the points of maximum (and minimum) argument of the regions $W_{0}(r)$ and $\tilde{W}(r)$ coincide. To see that the bound is not sharp for any $r>1 / \sqrt{2}$, we have only to observe that the extremal function $g$ for (3) maps $\mathbf{D}$ onto the complement of an analytic arc, while each extremal function $f$ for (2) has 
a range $\mathbf{C}-\Gamma$, where $\Gamma$ is a system of analytic arcs with a fork or a corner. (See Theorem B, case (iii).) But the proof of Theorem 1 shows $g(r)=\left(1-r^{2}\right) f^{\prime}(r)$ for $f=(g-1) / g^{\prime}(0) \in S$, so $\arg f^{\prime}(r)$ is maximal if $\arg g(r)=\pi+\log \left(r^{2} /\left(1-r^{2}\right)\right)$. Since the relation $f=(g-1) / g^{\prime}(0)$ would imply that $\mathbf{C}-f(\mathbf{D})$ is an analytic arc, this is impossible. Thus the bound is never sharp if $r>1 / \sqrt{2}$.

4. Proof of distinctness. We now show that $\mathcal{L}_{0}(r)$ is a proper subset of $\tilde{\mathcal{L}}(r)$. In fact, it will turn out that the boundaries of the two regions touch only at the four common points already identified. In particular, $W_{0}(r) \varsubsetneqq \tilde{W}(r)$.

THEOREM 2. $\mathcal{L}_{0}(r) \neq \tilde{\mathcal{L}}(r), 0<r<1$.

PROOF. If the two regions were identical, they would have the same support points. By the proof of Theorem 1, each function $g \in S_{0}$ which maximizes $\operatorname{Re}\left\{e^{i \sigma} \log h(r)\right\}$ must satisfy $g(r)=\left(1-r^{2}\right) f^{\prime}(r)$, where $f=(g-1) / g^{\prime}(0) \in S$. This function $f \in S$ solves the corresponding extremal problem (2) if and only if the boundary of $\mathcal{L}_{0}(r)$ touches that of $\tilde{\mathcal{L}}(r)$ in the direction $e^{-i \sigma}$. If this is the case, then a standard variational argument (see $[\mathbf{7}, \mathbf{6}]$ ) shows that $f$ maps $\mathbf{D}$ onto the complement of a system $\Gamma$ of analytic arcs which satisfy the differential equation

$$
\frac{e^{i \sigma} B(B-2 w)}{w^{2}(w-B)^{2}} d w^{2}>0, \quad B=f(r)
$$

In fact, $\Gamma$ consists of a single unbranched analytic arc unless $r>1 / \sqrt{2}$ and $e^{i \sigma}= \pm i$, as Theorem $B$ asserts.

On the other hand, the extremal function $g \in S_{0}$ for problem (3) maps $\mathbf{D}$ onto the complement of an analytic arc $\Gamma_{0}$ which extends from 0 to $\infty$ and satisfies (4). The relation $f=(g-1) / g^{\prime}(0)$ gives $g=1+g^{\prime}(0) f$. Because $g(z) \neq 0$, it follows that $\omega=-1 / g^{\prime}(0)$ is a value omitted by $f$. Thus $g=1-f / \omega$, where $\omega \in \Gamma$. But now it is clear that $\omega=w_{0}$, the finite tip of $\Gamma$, since $\Gamma_{0}$ terminates at the origin. In other words, $g=1-f / w_{0}$. This relation allows us to transform (4) into a second differential equation for $\Gamma$ :

$$
\frac{e^{i \sigma} B}{w\left(w-w_{0}\right)(B-w)} d w^{2}>0
$$

If $e^{i \sigma} \neq \pm 1$ or $\pm i$, then by Theorem $\mathrm{B}$ the arc $\Gamma$ has an analytic continuation to a full trajectory $\hat{\Gamma}$ of (7) which spirals into a double pole at either 0 or $B$. The extension $\hat{\Gamma}$ will not satisfy (8), because this quadratic differential has a simple pole at $w_{0}$, and so the trajectory terminates there. However, $\hat{\Gamma}-\Gamma$ is an orthogonal trajectory of (8), meaning that the quadratic differential is negative there. To see this, introduce a local analytic parametrization $w=\gamma(t)$ of $\hat{\Gamma}$ and note that the function of $t$ induced by (8) is analytic on an interval and real on half of the interval, so it must be real on the whole interval. (See for instance [5] for a more detailed explanation of this principle.)

Thus both expressions (7) and (8) are real on $\hat{\Gamma}$. Dividing (7) by (8) and taking the imaginary part, we obtain an algebraic expression for $\hat{\Gamma}$ :

$$
\operatorname{Im}\left\{\frac{(B-2 w)\left(w-w_{0}\right)}{w(w-B)}\right\}=0, \quad w \in \hat{\Gamma} .
$$


In other words, $\hat{\Gamma}$ is an algebraic curve satisfying an equation $P(u, v)=0$, where $w=u+i v$ and $P(u, v)$ is a polynomial in $u$ and $v$. But this is clearly impossible. Indeed, recall that $\hat{\Gamma}$ spirals into either 0 or $B$. (See Theorem B, case (ii).) This geometric property implies that the polynomial $P(u, 0)$ or $P(u, \operatorname{Im}\{B\})$ has infinitely many roots.

This contradiction shows that the regions $\mathcal{L}_{0}(r)$ and $\tilde{\mathcal{L}}(r)$ do not coincide for any value of $r$, and that they have no boundary points in common except for those of maximum and minimum real and (for $r \leq 1 / \sqrt{2}$ ) imaginary parts.

ACKNOWLEDGEMENT. The authors wish to thank Glenn Schober and Donald Wilken for helpful discussions while this research was in progress.

\section{REFERENCES}

1. L. V. Ahlfors, Complex analysis, 3rd ed., McGraw-Hill, New York, 1979.

2. I. A. Aleksandrov, Parametric extensions in the theory of univalent functions, Izdat. "Nauka", Moscow, 1976. (Russian)

3. I. A. Aleksandrov and S. A. Kopanev, The region of values of the derivative in the class of holomorphic univalent functions, Ukrain. Mat. Ž. 22 (1970), 660-664; English transl., Ukrainian Math. J. 22 (1970), 565-569.

4. Bateman Manuscript Project (A. Erdélyi, W. Magnus, F. Oberhettinger, F. Tricomi), Higher transcendental functions, Vol. II, McGraw-Hill, New York, 1953.

5. L. Brickman and St. Ruscheweyh, On certain support points of the class $S$, Proc. Amer. Math. Soc. 92 (1984), 61-63.

6. P. L. Duren, Arcs omitted by support points of univalent functions, Comment. Math. Helv. 56 (1981), 352-365.

7. _ Univalent functions, Springer-Verlag, Heidelberg and New York, 1983.

8. P. Duren and G. Schober, Nonvanishing univalent functions, Math. Z. 170 (1980), 195216.

9. $\_$, Nonvanishing univalent functions. II, Ann. Univ. Mariae Curie-Skłodowska 36/37 (1982/83), 33-43.

10. A. Grad, The region of values of the derivative of a schlicht function, in Coefficient Regions for Schlicht Functions by A. C. Schaeffer and D. C. Spencer, Amer. Math. Soc. Colloq. Publ., vol. 35, Amer. Math. Soc., Providence, R. I., 1950, Chapter XV.

11. D. Hamilton, Extremal problems for non-vanishing univalent functions, Aspects of Contemporary Complex Analysis (D. A. Brannan and J. G. Clunie, eds.), Academic Press, London, 1980, pp. 415-420.

12.

13. W. K. Hayman, Multivalent functions, Cambridge Univ. Press, London, 1958.

14. J. A. Jenkins, On univalent functions omitting two values, Complex Variables Theory Appl. 3 (1983), 169-172.

15. Z. Lewandowski, R. Libera and E. Złotkiewicz, Values assumed by Gelfer functions, Ann. Univ. Mariae Curie-Skłodowska 31 (1977), 75-84.

16. A. Pfluger, The range of $\log f^{\prime}(a)$, informal manuscript, 1982.

17. A. Ünal, Derivative type support points of the class $S, \mathrm{Ph} . \mathrm{D}$. Thesis, University of Michigan, 1986.

Department of Mathematics, University of Michigan, Ann ARbor, Michigan 48109

Department of Mathematics, University of Michigan, Flint, Michigan 48503 